\section{The Hippo signaling pathway restricts the oncogenic potential of an intestinal regeneration program}

\author{
Jing Cai, ${ }^{1}$ Nailing Zhang, ${ }^{1}$ Yonggang Zheng, ${ }^{1}$ \\ Roeland F. de Wilde, ${ }^{2}$ Anirban Maitra, ${ }^{2}$ \\ and Duojia Pan ${ }^{1,3}$
}

${ }^{1}$ Department of Molecular Biology and Genetics, Howard Hughes Medical Institute, Johns Hopkins University School of Medicine, Baltimore, Maryland 21205, USA; ${ }^{2}$ Department of Pathology, The Sol Goldman Pancreatic Cancer Research Center, Johns Hopkins University School of Medicine, Baltimore, Maryland 21205, USA

Although a developmental role for Hippo signaling in organ size control is well appreciated, how this pathway functions in tissue regeneration is largely unknown. Here we address this issue using a dextran sodium sulfate (DSS)-induced colonic regeneration model. We find that regenerating crypts express elevated Yes-associated protein (YAP) levels. Inactivation of YAP causes no obvious intestinal defects under normal homeostasis, but severely impairs DSS-induced intestinal regeneration. Conversely, hyperactivation of YAP results in widespread early-onset polyp formation following DSS treatment. Thus, the YAP oncoprotein must be exquisitely controlled in tissue regeneration to allow compensatory proliferation and prevent the intrinsic oncogenic potential of a tissue regeneration program.

Supplemental material is available at http://www.genesdev.org.

Received August 5, 2010; revised version accepted September 14, 2010.

Injury to many mammalian tissues triggers a sophisticated repair process that ultimately replenishes the lost cells and restores the structural and functional integrity of the affected tissues (Schafer and Werner 2008; Oviedo and Beane 2009). Such injury-induced cell proliferation must be tightly controlled to ensure that an appropriate number of cells are produced. Insufficient regenerative proliferation underlies pathological conditions such as age-related tissue atrophy and deterioration. Conversely, excessive and persistent regenerative proliferation may lead to tumorigenesis (Beachy et al. 2004; Kluwe et al. 2009). While a number of signaling molecules have been implicated in driving cell proliferation during tissue regeneration, little is known about mechanisms that normally restrict and/or terminate regenerative proliferation.

[Keywords: Hippo signaling; cancer; growth control; mouse; regeneration] ${ }^{3}$ Corresponding author.

E-MAIL djpan@jhmi.edu; FAX (410) 502-3177.

Article is online at http://www.genesdev.org/cgi/doi/10.1101/gad.1978810.
We reasoned that developmental pathways that normally terminate organ growth during animal development may also play a critical role in restricting regenerative growth in adult tissues. A prominent candidate is the Hippo tumor suppressor pathway (Badouel et al. 2009; Zhao et al. 2010). First identified in Drosophila, the Hippo pathway has emerged recently as a conserved mechanism that restricts organ size in diverse species, including mammals. This pathway comprises several tumor suppressors acting in a kinase cascade that culminates in the phosphorylation and inactivation of the transcriptional coactivator Yorkie (Yki) in Drosophila, or its mammalian homolog, YAP (Yes-associated protein) (Pan 2007; Zhao et al. 2010). In Drosophila and mice, inactivation of the tumor suppressors of the Hippo pathway or activation of the oncogene Yki or YAP results in massive tissue overgrowth characterized by increased cell proliferation and diminished cell death (Dong et al. 2007; Camargo et al. 2007; Zhou et al. 2009; Lee et al. 2010; Lu et al. 2010; Song et al. 2010). Conversely, inactivation of Yki or YAP leads to tissue and/or cellular atrophy (Huang et al. 2005; Zhang et al. 2010). While these findings demonstrate a critical role for Hippo signaling in controlling organ size during animal development and normal homeostasis, whether and how the Hippo pathway functions in the context of tissue regeneration are largely unknown.

\section{Results and Discussion}

To examine the role of Hippo signaling during tissue regeneration, we took advantage of the well-established dextran sodium sulfate (DSS)-induced colitis and regeneration model (Okayasu et al. 1990). A 5-d DSS treatment resulted in damages in the colonic crypt base and reduced the number of proliferating cells in the lower portion of the crypt (Fig. 1A,B). Two days after the withdrawal of DSS, the crypts were composed of tightly compacted proliferating cells that extended to the entire crypt, with a concomitant loss of differentiated goblet and enteroendocrine cells. Four days after DSS withdrawal, crypt histology and cell differentiation were largely restored, and cell proliferation was again restricted to the lower portion of the crypt (Fig. 1A,B,E). As shown previously, DSS-induced injury and regeneration was accompanied by induction of Stat3 phosphorylation (Bollrath et al. 2009; Grivennikov et al. 2009; Pickert et al. 2009), which was detected specifically in the damaged and regenerating crypts (Fig. 1D). We examined the temporal and spatial regulation of YAP in the DSS model. In normal adult mice, YAP protein is expressed in the entire crypt, including both proliferating and post-mitotic cells (Fig. 1C). The YAP protein level was slightly decreased in the crypts after a 5-d DSS treatment. Strikingly, a dramatic increase of YAP protein level was detected in the crypts $2 \mathrm{~d}$ after DSS withdrawal (Fig. 1C,D). This increase of YAP protein level was not due to increased transcription, since Yap mRNA was slightly decreased in the regenerating crypts (Fig. 1E). Despite the dramatic increase in YAP protein levels, the relative phosphorylation of YAP at its Hipporesponsive S112 site (as measured by P-S112-YAP/YAP ratio) was only slightly decreased in regenerating crypts (Fig. 1D), suggesting that Hippo signaling remained largely unperturbed in regenerating crypts. Consistent 


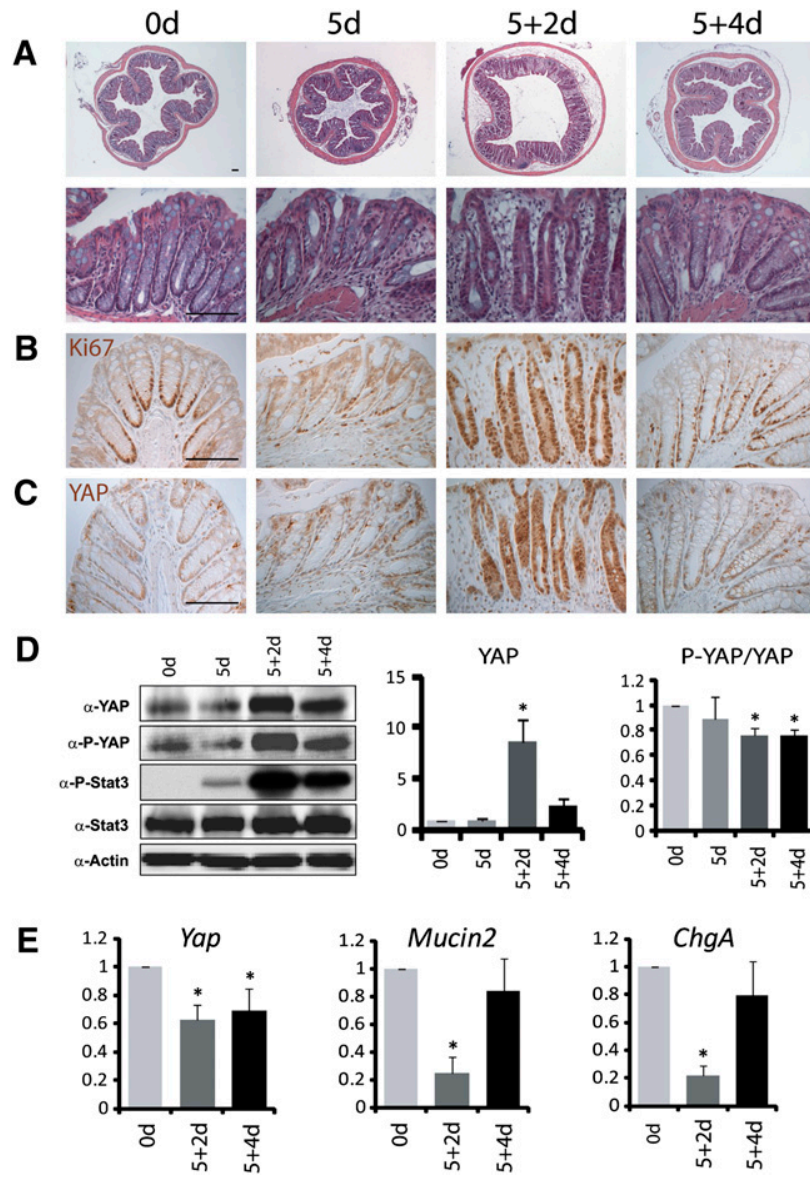

Figure 1. Increased YAP protein levels in regenerating crypts. $(A)$ H\&E staining of colon sections from 8-wk-old wild-type mice before $(0 \mathrm{~d})$ and after a 5-d DSS treatment (5 d), followed by normal drinking water for $2 \mathrm{~d}(5+2 \mathrm{~d})$ or $4 \mathrm{~d}(5+4 \mathrm{~d})$. (Top row) Low magnification. (Bottom row) High magnification. (B) Ki67 staining. Note the restriction of Ki67-positive proliferating cells to the crypt base in a normal colon, the loss of Ki67-positive cells in 5-d crypts, the expansion of Ki67-positive cells from the crypt base to the whole regenerating crypt in $5+2$-d colons, and the restoration of Ki67positive cells to the crypt base in $5+4-d$ colons. $(C)$ YAP staining. In wild-type crypts, YAP was detected in the entire crypt epithelium. Note the dramatic increase of YAP staining in $5+2$-d crypts. Also note the absence of appreciable YAP nuclear accumulation in control and regenerating crypts. $(D)$ Western blotting analysis. Protein extracts from control and regenerating crypts were probed with the indicated antibodies. Note the increase of YAP, P-YAP, and P-Stat3 in $5+2-d$ and $5+4-d$ crypts. The YAP protein level and $\mathrm{P}-\mathrm{YAP} / \mathrm{YAP}$ ratio were quantified in the graphs to the right. Data are mean \pm SD. $n=3 .\left(^{*}\right) P<0.01, t$-test. $(E)$ Real-time PCR analysis. mRNAs from control and regenerating crypts were analyzed for the expression of the indicated genes. Note the decrease of Yap mRNA in $5+2$-d and $5+4-d$ crypts. Also note the decreased expression of the goblet cell marker Mucin2 and the enteroendocrine cell marker Chromogranin A $(\mathrm{Chg} A)$ in $5+2$-d crypts, and the recovery of their expression levels in $5+4$-d crypts. Data are mean \pm SD. $n=3 .\left({ }^{*}\right) P<$ $0.01, t$-test. Bars, $100 \mu \mathrm{m}$.

with this finding, immunostaining showed that YAP was distributed nondiscriminatively in the cytoplasm and the nucleus in the regenerating crypts (Fig. 1C).

The dramatic increase in YAP protein levels in regenerating crypts suggests that YAP may play a potential role in intestinal regeneration. To investigate this possibility, we crossed a conditional knockout allele of Yap with
Villin-Cre (VilCre), which results in gene deletion in the epithelium of the small intestine and colon starting at embryonic day 12.5 (E12.5) (Madison et al. 2002). VilCre; Yap flox flox mice developed normally, and histological analysis of adult mice revealed no visible defects in cell differentiation, cell death, cell proliferation, or cell migration along the crypt-villus axis (Fig. 3; Supplemental Figs. S1, S3D,E), suggesting that YAP is dispensable for normal intestinal homeostasis. Following DSS treatment, however, VilCre; Yap flox flox mice showed a dramatic increase in mortality rate and a rapid decrease in body weight compared with the control littermates (Fig. 2A). Histological analysis revealed substantial damage with significant loss of crypts and scattered colonic epithelial cells in the VilCre; Yap flox flox mice compared with the control littermates (Fig. 2B,C). Furthermore, the VilCre; Yap flox flox colon contained fewer proliferating cells and more apoptotic cells (Fig. 2D,E). Thus, while YAP is largely dispensable for intestinal homeostasis under normal conditions, it is required for DSS-induced crypt regeneration.

The inconsequentiality of YAP loss for normal intestinal homeostasis can be compatible with at least two possibilities: the Hippo pathway may be simply dispensable, or, alternatively, Hippo signaling may be constitutively activated such that YAP is kept largely inactive under normal
A
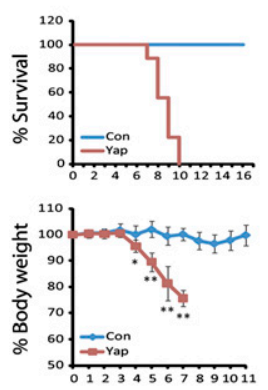

C

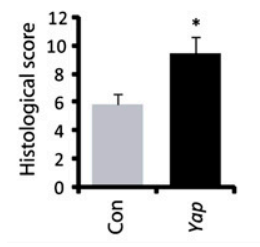

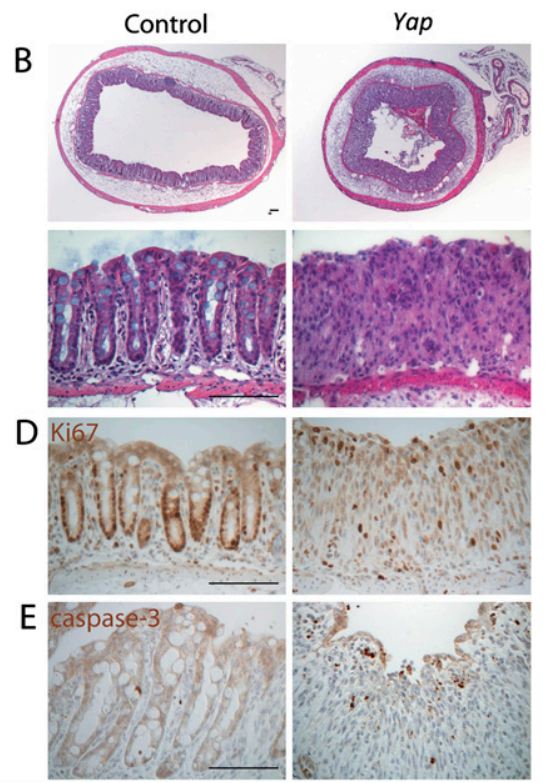

Figure 2. Impaired regeneration of Yap-deficient colonic crypts. $(A)$ Increased mortality and loss of body weight in Yap-deficient mice after DSS treatment. Mice were treated with $2.5 \%$ DSS for $7 \mathrm{~d}$ and supplied with normal drinking water thereafter. Thirteen wild-type and nine Yap-deficient mice were treated for mortality rate analysis. Nine wildtype and eight Yap-deficient mice were treated for body weight analysis. $\left(^{\star}\right) P<0.05$; $\left(^{\star \star}\right) P<0.01, t$-test. $(B)$ H\&E staining of colon sections after a 7-d DSS treatment. (Top row) Low magnification. (Bottom row) High magnification. Note the absence of crypt structure and the expansion of stromal cells in Yap-deficient colons. (C) Histological score of colons after a 7-d DSS treatment. Tissue damages were quantified as described in the Materials and Methods. Eight wild-type and nine Yap-deficient mice were treated for this analysis. Data are mean \pm SEM. $\left({ }^{*}\right) P<0.05$, $t$-test. $(D, E) \mathrm{Ki} 67$ and cleaved caspase-3 staining showing fewer proliferating and more apoptotic cells in Yap-deficient crypts after DSS treatment. Bars, $100 \mu \mathrm{m}$. 
homeostasis. The latter, but not the former, model predicts that inactivation of tumor suppressors that normally restrict YAP activity may lead to tumor formation and/or intestinal overgrowth due to hyperactivation of the YAP oncoprotein. To investigate this possibility, we generated conditional knockout mice for the Hippo pathway component Sav1 (Supplemental Fig. S2). Consistent with the latter model, 4-wk-old VilCre; Sav $1^{\text {flox }} /$ flox mice showed an enlargement of crypts in both the colon and small intestine, which contained more and faster proliferating cells (Fig. 3A-C; Supplemental Fig. S3). Interestingly, the hyperplasia of Sav1-deficient crypts was completely reversed by loss of YAP, even though the latter by itself had no visible effect on normal crypt morphology or cell proliferation (Fig. 3A-C; Supplemental Fig. S3). Consistent with the genetic suppression of the Sav1-deficient phenotype by loss of Yap, Sav1-deficient crypts showed decreased YAP S112 phosphorylation (Fig. 3D) as well as
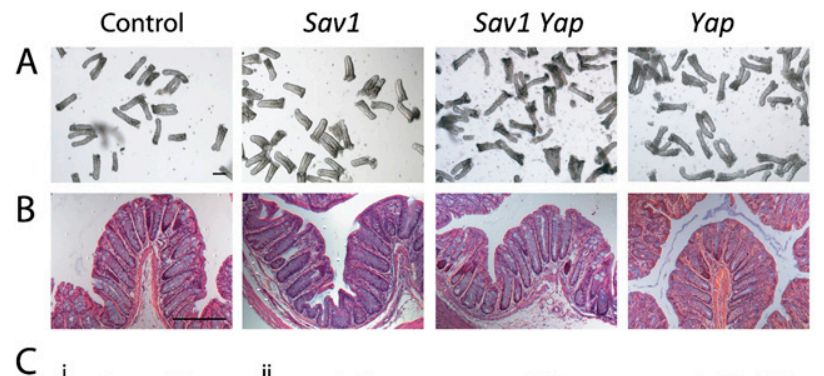

C
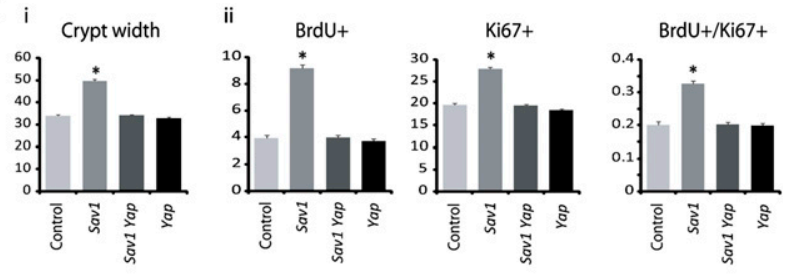

$\mathrm{D}$
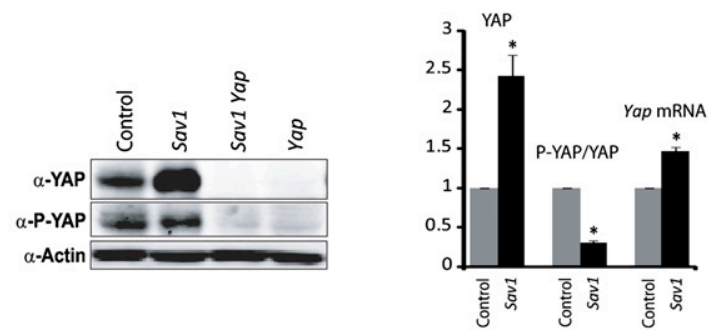

Figure 3. Loss of Sav1 results in Yap-dependent crypt hyperplasia. (A) Isolated colonic crypts from 4-wk-old wild-type, Sav1, Yap, or Sav1 Yap double-mutant colons. Note the enlarged width of Sav1 mutant crypts. Quantification of crypt width is shown in $C$, graph $i$. (B) H\&E staining of the colonic sections from 4-wk-old wild-type, Sav1, Yap, or Sav1 Yap double-mutant colons. Note the enlarged width of Sav1 mutant crypts. (C, graph i) Quantification of crypt width from $A$. Eighty crypts from three mice of each genotype were used. Data are mean \pm SEM. $\left(^{*}\right) P<0.01$, $t$-test. (Graph ii) Quantification of cell proliferation. Four-week-old wild-type, Sav1, Yap, or Sav1 Yap double-mutant mice were analyzed for BrdU and Ki67 staining $2 \mathrm{~h}$ after a single i.p. injection of BrdU. The number of $\mathrm{BrdU}^{+}$or $\mathrm{Ki} 7^{+}$cells and the ratio of $\mathrm{BrdU}^{+} / \mathrm{Ki} 67^{+}$in each crypt were quantified with 200 crypts from three mice of each genotype. Data are mean \pm SEM. $\left({ }^{\star}\right) P<0.01, t$-test. $(D)$ Quantification of YAP protein and mRNA levels. Note the increased YAP protein and mRNA levels in Sav1-deficient crypts. Also note that, while the absolute amount of P-YAP was similar in wild-type and Sav1deficient crypts, the P-YAP/YAP ratio was decreased in Sav1-deficient crypts. Data used in the graph are mean \pm SD. $n=3 .\left(^{\star}\right) P<0.01$, t-test. Bars, $100 \mu \mathrm{m}$. nuclear accumulation of YAP (Supplemental Fig. S2E), in contrast to its nondiscriminative subcellular localization in normal or regenerating crypts (Fig. 1C). By 13 mo of age, all male VilCre; Sav1 $1^{\text {flox }}$ flox mice had developed colonic polyps (average number $1.71 \pm 0.76, n=7$ ), whereas none of the control male littermates developed any polyps at a comparable age $(n=12)$ (Fig. 4A). Histological analysis revealed that the polyps did not resemble the prototypal intestinal adenomas observed in the $A p c^{M i n}$ model of tumorigenesis (Moser et al. 1990; Su et al. 1992). In contrast, there were prominent invaginations of the hyperplastic epithelium leading to a "saw-tooth" appearance of the glands, a feature characteristic of sessile serrated polyps (SSPs), which comprise a recently catalogued alternative pathway to colorectal neoplasia in humans (Fig. 4B, panel i; Montgomery 2004; Snover et al. 2005). Over time, SSPs left in situ are prone to adenomatous transformation and the development of invasive adenocarcinomas (Montgomery 2004; Snover et al. 2005). Indeed, such an adenomatous transformation (Fig. 4B, panel ii) and invasion of the muscularis mucosa by neoplastic glands (Fig. 4B, panel iii) were observed. The Sav1-deficient SSPs showed nuclear accumulation of YAP, but not nuclear accumulation of $\beta$-catenin or elevated overall levels of $\beta$-catenin (Fig. 4D,E), further underscoring their distinction from the conventional tubular adenomas. Consistent with the complete suppression of Sav1 lossinduced crypt hyperplasia by loss of YAP in younger mice, none of the aged VilCre;Sav1 $1^{\text {flox }}{ }^{\text {flox }}$; Yap flox flox $(n=6$ males) and VilCre; Yap flox flox mice ( $n=6$ males) developed any polyps or histological abnormalities in the colon. To our knowledge, the VilCre;Sav1 flox flox mice described here represent the first mouse model for SSPs.

The development of SSPs in Sav1-deficient mouse colons prompted us to investigate whether aberrant Hippo signaling may be a general hallmark of SSPs in humans. Therefore, we examined the expression and subcellular localization of YAP and $\beta$-catenin proteins in an archival collection of 14 histologically documented SSPs that had been endoscopically removed (Fig. 4F-J). All of the 14 SSPs $(100 \%)$ demonstrated up-regulation of YAP protein, compared with the adjacent normal colonic mucosa (Fig. 4F,H). Eleven of $14(78 \%)$ SSPs demonstrated unequivocal nuclear YAP accumulation within the polypoid epithelium, while three of $14(22 \%)$ demonstrated only infrequent nuclear YAP labeling, although cytoplasmic protein was still up-regulated compared with the colonic epithelium. Of note, in the 11 SSPs with nuclear YAP localization, we confirmed the absence of nuclear $\beta$-catenin in serial sections of the polypoid epithelium, and its restriction to the membranous and cytoplasmic compartments (Fig. 4I,J). Thus, as in Sav1-deficient mouse colons (Fig. 4D,E), nuclear localization of YAP occurs in the absence of nuclear or overall $\beta$-catenin accumulation in human SSPs.

The dispensable role for Yap but not Sav1 in normal intestinal growth is consistent with the view that, under normal homeostasis, the Hippo pathway keeps the YAP oncoprotein in a relatively inactive state. This raises the critical question of why intestinal cells invest the energy to synthesize a growth regulator like YAP, but meanwhile employ an elaborate signaling cascade to turn off its activity. An attractive model is that the intestinal epithelia, which are constantly exposed to environmental insults, must be poised for regeneration in anticipation of severe tissue damage. This regenerative capacity, which is 
Cai et al.
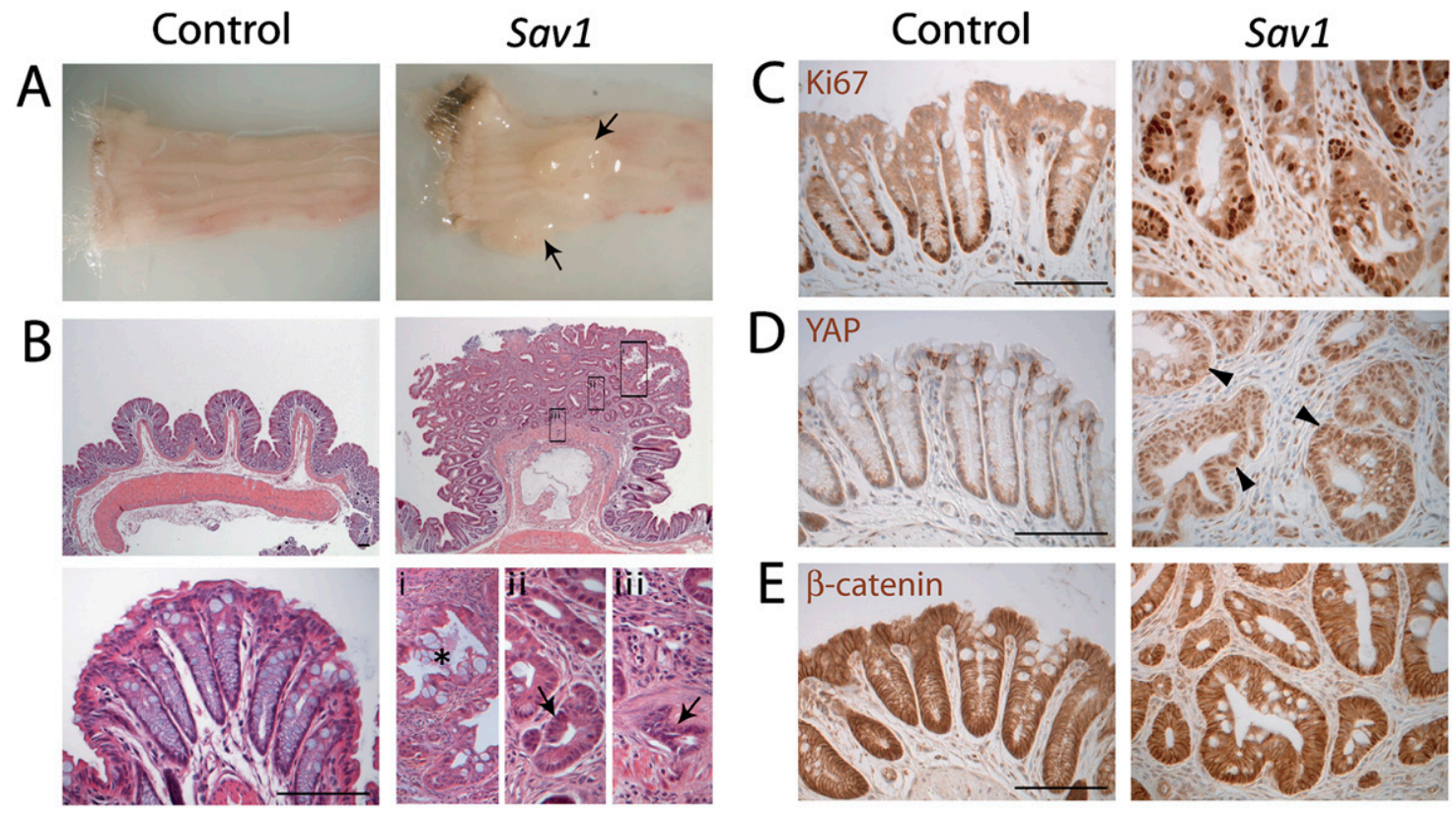

$\mathrm{F}$

$\begin{array}{ccccc}\begin{array}{c}\text { SSP } \\ \text { case }\end{array} & \begin{array}{c}\text { YAP } \\ \text { cytoplasmic }\end{array} & \begin{array}{c}\text { YAP } \\ \text { nuclear }\end{array} & \begin{array}{c}\beta \text {-catenin } \\ \text { cytoplasmic }\end{array} & \begin{array}{c}\beta \text {-catenin } \\ \text { nuclear }\end{array} \\ 1 & ++ & + & + & - \\ 2 & ++ & ++ & + & - \\ 3 & + & +/- & + & - \\ 4 & + & + & + & - \\ 5 & + & + & + & - \\ 6 & + & +/- & + & - \\ 7 & + & + & + & - \\ 8 & + & + & + & - \\ 9 & + & + & + & - \\ 10 & + & +/- & + & - \\ 11 & + & + & + & - \\ 12 & + & + & + & - \\ 13 & ++ & ++ & + & - \\ 14 & + & + & + & -\end{array}$
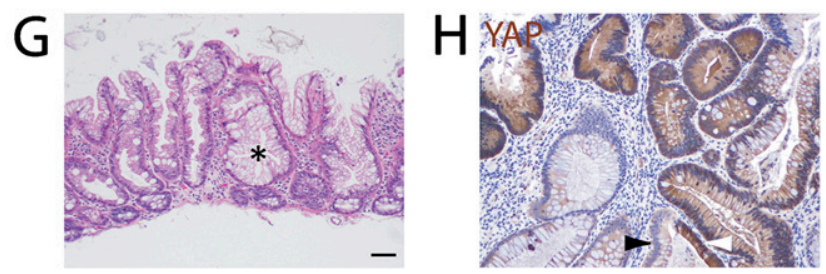

I
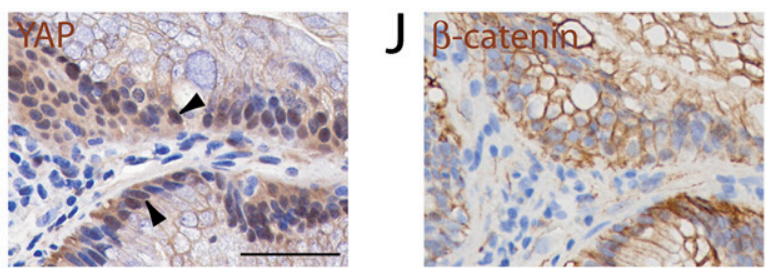

Figure 4. SSP development in Sav1-deficient mouse colons and the prevalence of Hippo pathway dysregulation in human SSPs. (A) Distal colon of 13-mo-old wild-type control and Sav1-deficient littermates. Note the presence of two polyps in the Sav1-deficient colon (black arrows). (B) H\&E staining of the colonic sections from 13-mo-old wild-type control (left) and Sav1-deficient (right) littermates. The top and bottom panels show the corresponding low- and high-magnification images, respectively. Note the presence of serrated polyps with "saw-tooth" crypt epithelium (asterisk in panel i), adenomatous transformation (arrow in panel ii), and invasion of the muscularis mucosa (arrow in panel iii) in Sav1-deficient colons. $(C)$ Ki67 staining. Note that Ki67-positive proliferating cells were restricted to the crypt base in control colons. In Sav1deficient colons, scattered Ki67-positive cells were detected throughout the crypt epithelia. $(D, E)$ YAP and $\beta$-catenin staining. Note the accumulation of nuclear YAP but not nuclear $\beta$-catenin in Sav1-deficient colonic polyps (arrows). (F- $J$ ) Analysis of human SSPs. (F) Summary of YAP and $\beta$-catenin staining in human SSPs. $(G)$ Histopathology of a right-sided SSP obtained by endoscopic excision. H\&E stain, 10X magnification. Note the characteristic polypoid epithelium. $(H)$ Up-regulation of YAP in the polypoid epithelium of an SSP compared with adjacent normal colonic epithelium. At the bottom right half of the panel, one gland was partially involved by SSP and demonstrated YAP upregulation (white arrowhead), while the nonneoplastic half of the gland had minimal expression (black arrowhead). (I) High-power magnification of SSP epithelium with nuclear YAP labeling. Magnification, 40×. (J) Absence of nuclear $\beta$-catenin, with retained membranous localization of the protein, in the serial section of the SSP shown in D. Magnification, $40 \times$. Bars, $100 \mu \mathrm{m}$.

YAP-dependent, must be kept in check by the Hippo pathway to prevent excessive proliferation. Our observation that loss of Sav1 leads to crypt hyperplasia and tumorigenesis under normal homeostasis is consistent with this model. We further reasoned that Hippo-mediated suppression of YAP may be even more critical for preventing tumorigenesis during DSS-induced intestinal repair, when regenerating crypts express highly elevated levels of the YAP oncoprotein. To test this hypothesis, we treated 12-wk-old wild-type control, VilCre;Sav1 flox flox, VilCre; Sav $1^{\text {flox }}$ flox $_{\text {; Yap }}$ flox fllox , and VilCre; Yap flox flox mice with DSS for $4 \mathrm{~d}$ and allowed them to grow for 3 mo with normal drinking water. Strikingly, all VilCre; Sav $1^{\text {flox flox }}$ mice developed multiple colonic polyps by 3 mo $(n=7)$, whereas none of the age- and treatment-matched control littermates developed colonic polyps $(n=8)$, nor did any VilCre;Sav $1^{\text {flox } / \text { flox }}$ mice without DSS treatment $(n=8)$ at a comparable age (Fig. 5A). In fact, the latter did not develop colonic polyps before 12 mo of age. This enhanced tumorigenicity is YAP dependent, as none of the age- and treatment-matched VilCre; Sav1 flox fflox; Yap $^{\text {flox }}$ flox $(n=5)$ or VilCre; Yap flox fllox $(n=5)$ mice developed 


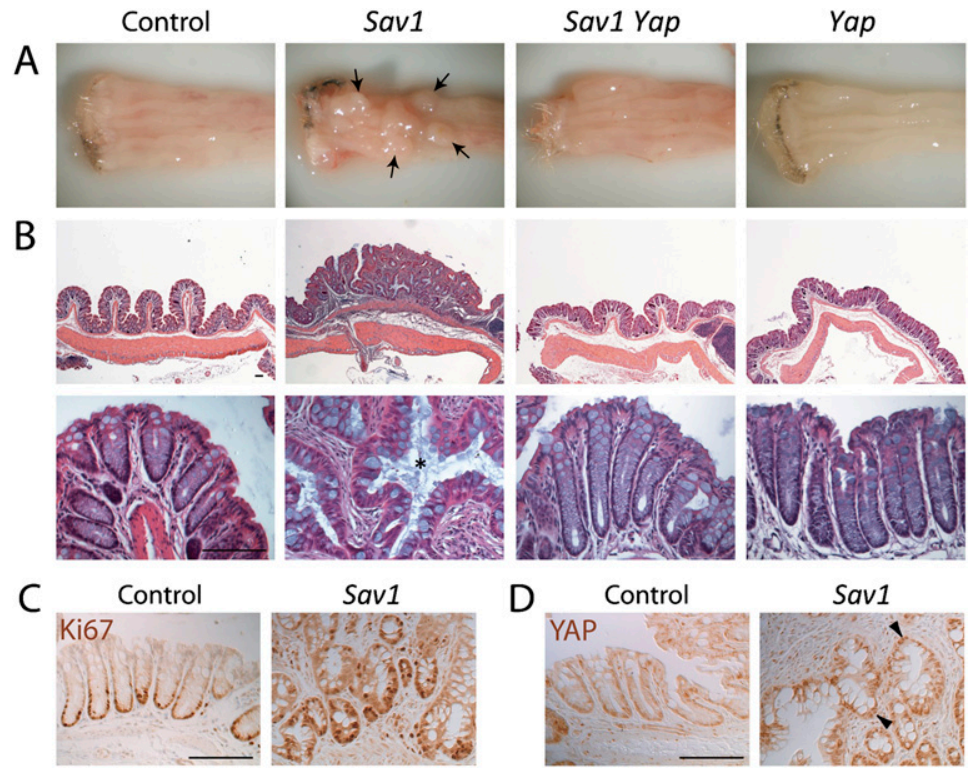

Figure 5. DSS-induced regeneration accelerated polyp development in Sav1deficient colons in a Yap-dependent manner. (A) Distal colon of 12-wk-old wildtype, Sav1, Yap, or Sav1 Yap double-mutant mice treated with 2.5\% DSS for 4 d, followed by normal drinking water for $3 \mathrm{mo}$. Note the presence of multiple large colonic polyps in the Sav1-deficient colon (arrows). (B) H\&E staining of colonic sections from animals in $A$. The top and bottom panels show the corresponding low- and high-magnification images, respectively. Note the presence of serrated crypt epithelium in Sav1-deficient polyps (asterisk). (C) Ki67 staining of colon sections from control and Sav1-deficient littermates. Note the presence of scattered Ki67-positive cells throughout the serrated crypt epithelium. $(D)$ YAP staining of colon sections from control and Sav1-deficient littermates. Note the accumulation of nuclear YAP in Sav1-deficient colonic polyps (arrowheads). Bars, $100 \mu \mathrm{m}$.

colonic polyps. Histological analysis and Ki67 staining revealed the presence of SSPs in DSS-induced Sav1-deficient colons (Fig. 5B,C). Like the late-onset polyps observed in Sav1-deficient colons without DSS treatment, the DSS-induced Sav1-deficient polyps showed an accumulation of nuclear YAP (Fig. 5D). Taken together, we conclude that DSS-induced injury and repair greatly exacerbated the tumorigenicity of the Sav1-deficient crypts.

The prevailing view that the Hippo pathway functions as a critical regulator of mammalian tissue homeostasis is based largely on the premise that YAP gain of function leads to tissue overgrowth and cell transformation (Overholtzer et al. 2006; Zender et al. 2006; Camargo et al. 2007; Dong et al. 2007; Zhou et al. 2009; Lee et al. 2010; Lu et al. 2010; Song et al. 2010). Whether YAP is normally required for mammalian tissue homeostasis is less clear. Our present study demonstrates that YAP is dispensable for normal intestinal homeostasis, but is required for intestinal regeneration following tissue injury. We note that the requirement for YAP in DSSinduced regeneration resembles that of Stat3 (Bollrath et al. 2009; Grivennikov et al. 2009; Pickert et al. 2009), suggesting a potential cross-talk between the Hippo and the JAK/STAT pathways. Since Stat3 phosphorylation was not affected in Yap- or Sav1-deficient crypts after DSS treatment (data not shown), it is unlikely that Stat3 functions downstream from the Hippo pathway. Whether the Hippo pathway resides downstream from Stat3 or functions in parallel to the JAK/Stat3 pathway requires further investigation.
Our current study also offers an example of a molecular explanation for the long-standing association between tissue regeneration and cancer (Beachy et al. 2004; Kluwe et al. 2009). Following DSS-induced injury, regenerating crypts express highly elevated levels of the YAP oncoprotein. Given YAP's well-established transforming activity, this regenerative response is potentially oncogenic, and therefore must be kept in check via Hippo-mediated YAP phosphorylation. Loss of Hippo signaling, as in the Sav1-deficient crypts, can expose the intrinsic oncogenic potential of this regeneration program and result in accelerated malignant transformation. It is tempting to speculate that other regenerative responses may share a similar oncogenic potential, and that the Hippo pathway may play a more widespread role in restraining the oncogenic potential intrinsic to these regenerative processes.

\section{Materials and methods}

\section{Generation of Sav1 conditional knockout mice}

A targeting vector containing the third exon of the Sav1 gene was generated by recombineering as described previously (Liu et al. 2003). Transformed embryonic stem cell colonies were screened by long-template PCR with the following primer sets: PS5F (5'-GAAAAACACCTCTCACCTCTGAATATCATTCCC TCCTCCAGCTCC-3') and PS5R (5'-TTAAGGGTTATTGAA TATGATCGGAATTGGGCTGCAGGAA-3') to generate a 4.4-kb band for positive clones; and PS3F (5'-GCTCTATGGCTTCT GAGGCGGAAAGAACCAGCTGGGGCTCGAC-3') and PS3R (5'-CTCAAGGAAACACAAGGTAGCTCATGAACTATCTCG TATTCAGGG-3') to generate a 4.8 -kb band for positive clones.

Successfully targeted embryonic stem cell clones (confirmed by both 5' PCR and 3' PCR) were microinjected into C57BL/6 blastocysts. Germline transmission from generated chimeric offspring was confirmed by long-template PCR (Supplemental Fig. S2A,B). Mice carrying the targeted allele were bred to Flp recombinase transgenic mice (kindly provided by Dr. Jeremy Nathans, Johns Hopkins University School of Medicine) to remove the FRT-flanked Neo cassette and to generate the Sav1 $1^{\text {flox }}$ mice.

Genomic DNAs extracted from tail biopsies were genotyped with a PCR primer set (PS1, 5' -AGGGGATTCTGACATTTCAGTCAGTT-3'; PS2, 5'AGTCACATGCTGACCACAAGCAGAA-3'; PS3; 5'-TGCCATTAAGTG TAATCACTGG-3') that generated a 231-base-pair (bp) band from the wildtype allele, a 335-bp band from the Flox allele, and a 460-bp band from the knockout allele (Supplemental Fig. S2C).

Yap ${ }^{\text {flox }}$ mice were generated as described (Zhang et al. 2010). Villin-Cre mice were purchased from the Jackson Laboratory. Mice with Sav1 or Yap specifically deleted in the intestinal epithelium were generated by breeding Sav $1^{\text {flox }}$ or Yap ${ }^{\text {flox }}$ mice with VilCre mice. The Cre, Yap knockout, and Yap Flox alleles were genotyped as described (Zhang et al. 2010). Animal protocols were approved by the Institutional Animal Care and Use Committee of Johns Hopkins University.

\section{DSS treatment of mice}

For induction of colitis, mice received 2.5\% DSS (MP Biomedical, molecular weight $36,000-50,000 \mathrm{kDa}$ ) in drinking water for several days as indicated in the text. After DSS treatment, mice were supplied with normal drinking water and euthanized, and colons were harvested at the time points indicated in the text.

\section{Isolation of intestinal epithelial cells}

Colons at $\sim 1 \mathrm{~cm}$ away from the anus were cut longitudinally and rinsed in PBS to remove feces. Pieces $(2-3 \mathrm{~mm})$ of colons were incubated in PBS 
with $5 \mathrm{mM}$ EDTA for $15 \mathrm{~min}$ at $37^{\circ} \mathrm{C}$. Vigorous shaking released crypts. Crypts were photographed and the width was measured in AxioVision release 4.7. For RNA and protein analysis, the supernatant containing free crypts was collected and centrifuged. The pellet was washed in ice-cold PBS and subjected to RNA extraction or snap-frozen in liquid nitrogen for Western blot analysis.

\section{Analysis of YAP protein expression in human SSPS}

Formalin-fixed, paraffin-embedded sections were obtained from 14 archival SSPs that had been removed endoscopically at Johns Hopkins Hospital. The diagnosis of SSP was rendered based on established criteria by a specialized gastrointestinal pathologist (A.M.) (Montgomery 2004; Snover et al. 2005). Heat-induced antigen retrieval was performed in a steamer using citrate buffer ( $\mathrm{pH}$ 6.0) (Vector Laboratories) for $25 \mathrm{~min}$ followed by $30 \mathrm{~min}$ of cooling. Nonspecific binding was blocked for $10 \mathrm{~min}$ with $5 \%$ bovine serum albumin (BSA) in TBST. Serial sections were then incubated with two primary antibodies-anti-YAP (1:150 dilution; catalog no. 2060-1, Epitomics Inc.) and anti- $\beta$-catenin (1:1000 dilution; catalog no. DS9800, $\mathrm{BD}$ Transduction)-for $1 \mathrm{~h}$ at room temperature. The sections were then incubated for $30 \mathrm{~min}$ with secondary antibody (Leica Microsystems) followed by detection with 3,3'-Diaminobenzide (Sigma-Adrich Co.) for $1 \mathrm{~min}$. Sections were washed three times after each step in Tris-buffered saline with $0.1 \%$ Tween-20. Finally, sections were counterstained with Harris hematoxylin, subsequently rehydrated in distilled $\mathrm{H}_{2} \mathrm{O}$ and graded series of ethanol $(70 \%, 95 \%$, and $100 \%)$, and mounted.

\section{Acknowledgments}

We thank Drs. Qian Chen, Karen K. David, and Jianzhong Yu for discussion. A.M. is supported by P01CA134292, R01CA113669, and R01CA134767. D.P. is an investigator of the Howard Hughes Medical Institute.

\section{References}

Badouel C, Garg A, McNeill H. 2009. Herding Hippos: Regulating growth in flies and man. Curr Opin Cell Biol 21: 837-843.

Beachy PA, Karhadkar SS, Berman DM. 2004. Tissue repair and stem cell renewal in carcinogenesis. Nature 432: 324-331.

Bollrath J, Phesse TJ, von Burstin VA, Putoczki T, Bennecke M, Bateman T, Nebelsiek T, Lundgren-May T, Canli O, Schwitalla S, et al. 2009. gp130-mediated Stat3 activation in enterocytes regulates cell survival and cell-cycle progression during colitis-associated tumorigenesis. Cancer Cell 15: 91-102.

Camargo FD, Gokhale S, Johnnidis JB, Fu D, Bell GW, Jaenisch R, Brummelkamp TR. 2007. YAP1 increases organ size and expands undifferentiated progenitor cells. Curr Biol 17: 2054-2060.

Dong J, Feldmann G, Huang J, Wu S, Zhang N, Comerford SA, Gayyed MF, Anders RA, Maitra A, Pan D. 2007. Elucidation of a universal sizecontrol mechanism in Drosophila and mammals. Cell 130: 1120-1133.

Grivennikov S, Karin E, Terzic J, Mucida D, Yu GY, Vallabhapurapu S, Scheller J, Rose-John S, Cheroutre H, Eckmann L, et al. 2009. IL-6 and Stat3 are required for survival of intestinal epithelial cells and development of colitis-associated cancer. Cancer Cell 15: 103-113.

Huang J, Wu S, Barrera J, Matthews K, Pan D. 2005. The Hippo signaling pathway coordinately regulates cell proliferation and apoptosis by inactivating Yorkie, the Drosophila homolog of YAP. Cell 122: 421-434.

Kluwe J, Mencin A, Schwabe RF. 2009. Toll-like receptors, wound healing, and carcinogenesis. J Mol Med 87: 125-138.

Lee KP, Lee JH, Kim TS, Kim TH, Park HD, Byun JS, Kim MC, Jeong WI, Calvisi DF, Kim JM, et al. 2010. The Hippo-Salvador pathway restrains hepatic oval cell proliferation, liver size, and liver tumorigenesis. Proc Natl Acad Sci 107: 8248-8253.

Liu P, Jenkins NA, Copeland NG. 2003. A highly efficient recombineering-based method for generating conditional knockout mutations. Genome Res 13: 476-484.

Lu L, Li Y, Kim SM, Bossuyt W, Liu P, Qiu Q, Wang Y, Halder G, Finegold MJ, Lee JS, et al. 2010. Hippo signaling is a potent in vivo growth and tumor suppressor pathway in the mammalian liver. Proc Natl Acad Sci 107: 1437-1442.
Madison BB, Dunbar L, Qiao XT, Braunstein K, Braunstein E, Gumucio DL. 2002. Cis elements of the villin gene control expression in restricted domains of the vertical (crypt) and horizontal (duodenum, cecum) axes of the intestine. J Biol Chem 277: 33275-33283.

Montgomery E. 2004. Serrated colorectal polyps: Emerging evidence suggests the need for a reappraisal. Adv Anat Pathol 11: 143-149.

Moser AR, Pitot HC, Dove WF. 1990. A dominant mutation that predisposes to multiple intestinal neoplasia in the mouse. Science 247: 322-324.

Okayasu I, Hatakeyama S, Yamada M, Ohkusa T, Inagaki Y, Nakaya R. 1990. A novel method in the induction of reliable experimental acute and chronic ulcerative colitis in mice. Gastroenterology 98: 694-702.

Overholtzer M, Zhang J, Smolen GA, Muir B, Li W, Sgroi DC, Deng CX, Brugge JS, Haber DA. 2006. Transforming properties of YAP, a candidate oncogene on the chromosome 11q22 amplicon. Proc Natl Acad Sci 103: 12405-12410.

Oviedo NJ, Beane WS. 2009. Regeneration: The origin of cancer or a possible cure? Semin Cell Dev Biol 20: 557-564.

Pan D. 2007. Hippo signaling in organ size control. Genes Dev 21: 886897.

Pickert G, Neufert C, Leppkes M, Zheng Y, Wittkopf N, Warntjen M, Lehr HA, Hirth S, Weigmann B, Wirtz S, et al. 2009. STAT3 links IL22 signaling in intestinal epithelial cells to mucosal wound healing. J Exp Med 206: 1465-1472.

Schafer M, Werner S. 2008. Cancer as an overhealing wound: An old hypothesis revisited. Nat Rev Mol Cell Biol 9: 628-638.

Snover DC, Jass JR, Fenoglio-Preiser C, Batts KP. 2005. Serrated polyps of the large intestine: A morphologic and molecular review of an evolving concept. Am I Clin Pathol 124: 380-391.

Song H, Mak KK, Topol L, Yun K, Hu J, Garrett L, Chen Y, Park O, Chang J, Simpson RM, et al. 2010. Mammalian Mst1 and Mst2 kinases play essential roles in organ size control and tumor suppression. Proc Natl Acad Sci 107: 1431-1436.

Su LK, Kinzler KW, Vogelstein B, Preisinger AC, Moser AR, Luongo C, Gould KA, Dove WF. 1992. Multiple intestinal neoplasia caused by a mutation in the murine homolog of the APC gene. Science 256: 668-670.

Zender L, Spector MS, Xue W, Flemming P, Cordon-Cardo C, Silke J, Fan ST, Luk JM, Wigler M, Hannon GJ, et al. 2006. Identification and validation of oncogenes in liver cancer using an integrative oncogenomic approach. Cell 125: 1253-1267.

Zhang N, Bai H, David KK, Dong J, Zheng Y, Cai J, Giovannini M, Liu P, Anders RA, Pan D. 2010. The Merlin/NF2 tumor suppressor functions through the YAP oncoprotein to regulate tissue homeostasis in mammals. Dev Cell 19: 27-38.

Zhao B, Li L, Lei Q, Guan KL. 2010. The Hippo-YAP pathway in organ size control and tumorigenesis: An updated version. Genes Dev 24: 862-874.

Zhou D, Conrad C, Xia F, Park JS, Payer B, Yin Y, Lauwers GY, Thasler W, Lee JT, Avruch J, et al. 2009. Mst1 and Mst2 maintain hepatocyte quiescence and suppress hepatocellular carcinoma development through inactivation of the Yap1 oncogene. Cancer Cell 16: 425-438. 


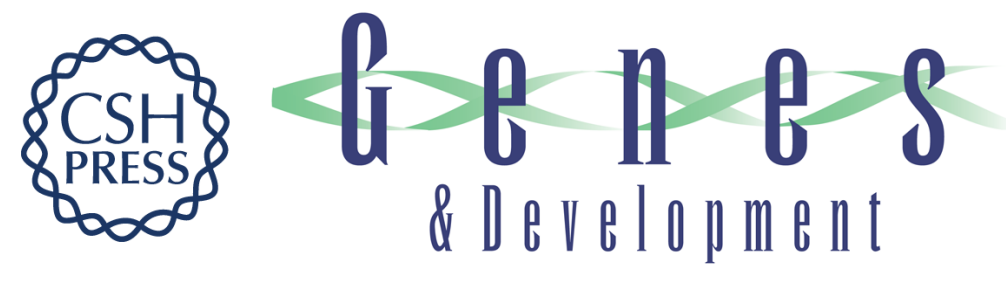

\section{The Hippo signaling pathway restricts the oncogenic potential of an intestinal regeneration program}

Jing Cai, Nailing Zhang, Yonggang Zheng, et al.

Genes Dev. 2010, 24:

Access the most recent version at doi:10.1101/gad.1978810

\section{Supplemental http://genesdev.cshlp.org/content/suppl/2010/10/21/24.21.2383.DC1 \\ Material}

Related Content

Hippo Checks Regeneration

Annalisa M. VanHook

Sci. Signal. November , 2010 3: ec342

References This article cites 27 articles, 11 of which can be accessed free at:

http://genesdev.cshlp.org/content/24/21/2383.full.html\#ref-list-1

Articles cited in:

http://genesdev.cshlp.org/content/24/21/2383.full.html\#related-urls

\section{License}

Email Alerting

Service

Receive free email alerts when new articles cite this article - sign up in the box at the top right corner of the article or click here.

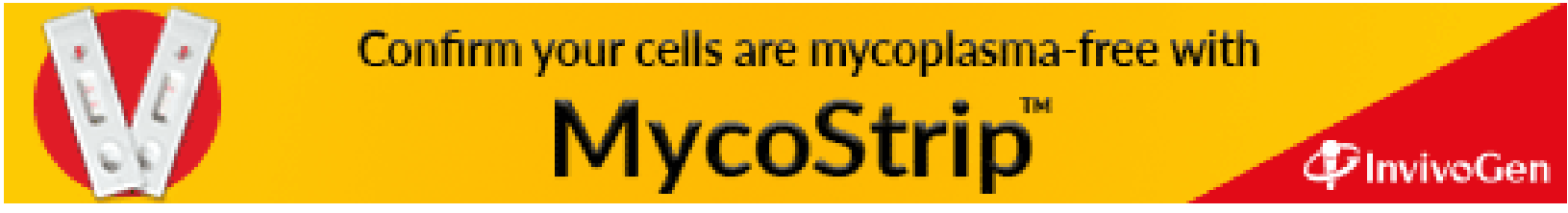

